\title{
XX. Beiträge zur Morphologie einiger künstlich dargestellter Verbindungen.
}

\author{
Von \\ V. Rosicky' in Prag. \\ (Mit 19 Textfiguren.)
}

Die vorliegende Arbeit wurde im mineralogischen Institute der $\mathbf{k}$. Universität in München ausgeführt.

Ich erfülle an dieser Stelle meine angenehme Pflicht, dem Director des Institutes, Herrn Geheimrat Prof. Dr. P. v. Groth, für die Überlassung des Materiales, sowie für das Interesse, das er dieser Arbeit gewidmet hat, meinen innigsten Dank auszusprechen.

\section{I. Über die isomorphe Reihe der Äthylendiaminsulfate von Eisen, Mangan und Cadmium.}

Die Salze von der Constitution:

$$
\mathrm{Me}\left(\mathrm{SO}_{4}\right)_{2} \underset{\mathrm{CH}_{2} \cdot \mathrm{NH}_{2}}{\mathrm{CH}}+\mathrm{NH}_{2}+4 \mathrm{aq},
$$

wo $M e=F^{\top} e, M n, C d$ ist, wurden von H. Großmann und B. Schück ${ }^{1}$ ) chemisch mit anderen analogen Verbindungen untersucht.

Dr. H. Steinmetz in München, welcher einige vorläufige Messungen der Salze vorgenommen hatte ${ }^{2}$ ), hatte mir die obenerwähnten Verbindungen zur definitiven goniometrischen Untersuchung übergeben.

Diese Salze krystallisieren triklin pinakoidal. In den Winkeln der Flächen nähern sich die Krystalle der höheren, trigonalen Symmetrie. Dieser Pseudosymmetrie entspricht auch in manchen Fällen die äußere

1) Zeitschr. f. anorgan. Chemie $1906,50,26-29$.

2) Ebenda. 
Ausbildung der Krystalle, indem dieselben einer trigonalen Combination recht ähnlich erscheinen, wie beim Eisen- und Mangansalze gezeigt werden wird.

Die pseudotrigonale Symmetrie wird besonders durch eine stereographische Projection der Combination eines Salzes klar gemacht, wie es die Fig. 1 für das Mangansalz darstellt 1). In der Projection sehen wir, daß Fig. 1.

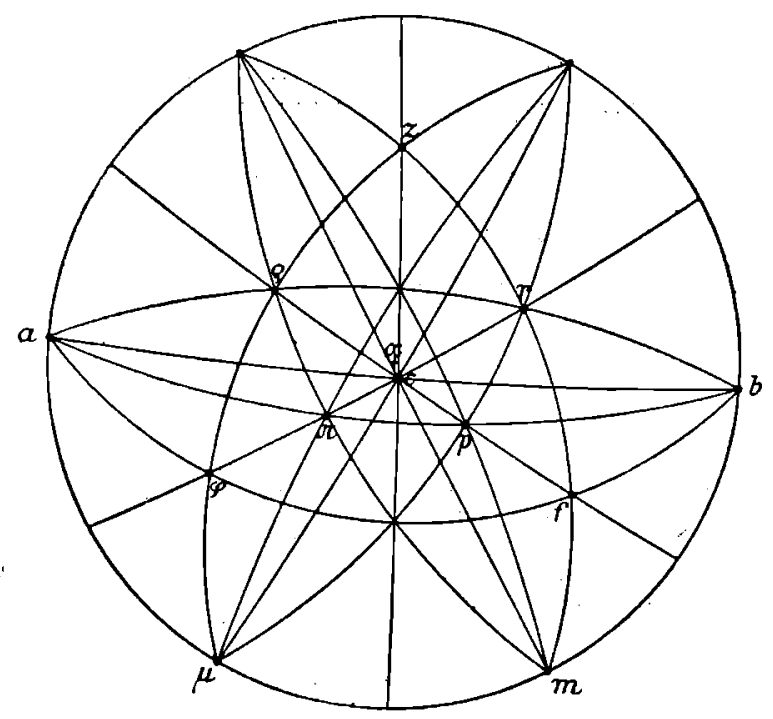

die Prismenflächen $m$ und $\mu$ mit den Brachypinakoidflächen $b$ ein hexagonales Deuteroprisma nachahmen, und die Formen $f, \varphi, p$, $\pi$, sowie $r$ und $\varrho$ positiven resp. negativen Rhomboëderflächen entsprechen. Demzufolge wurde auch den Krystallen eine dieser höheren Symmetrie entsprechende Aufstellung gegeben.

Keines der Glieder dieser Reihe zeigt eine Spaltbarkeit.

\section{Äthylendiaminferrosulfat-Tetrahydrat.}

Die Krystalle sind meist dicktafelig nach der Basis $e\{001\}$, seltener sind sie nach der verticalen Axe etwas gestreckt. Ihre Farbe ist hellgrünlich, ihr Glanz glasig.

Folgende Formen wurden beobachtet: $e\{001\}, b\{010\}, m\{110\}$, $\mu\{1 \overline{1} 0\}, d\{\overline{8} 03\}, p\{133\}, \pi\{1 \overline{3} 3\}, f\{4.12 .3\}, \varphi\{4 . \overline{1} \overline{2} .3\}, \varepsilon\{\overline{8} . \overline{2} \overline{4} .3\}$,

Die größeren Krystallflächen waren manchmal etwas gekrümmt oder durch Vicinalflächen ersetzt, so daß sie doppelte Signale lieferten. Doch

1) Statt $\approx$ lies $d$, Für die beiden anderen Salze wäre die Projection ganz ähnlich. 
konnte die dadurch bedingte Ungenauigkeit durch Reflexion der Gegenflächen in den meisten Fällen beseitigt werden. Die Flächen der verticalen Zone, der Basis und der Pyramiden $\tau$ und $p$ reflectierten meistens gut. Insgesamt wurden vier Krystalle durchgemessen.

Der Berechnung der Krystallelemente wurden folgende Winkel zugrunde gelegt:

$$
\begin{aligned}
& m: \mu=(110):(1 \overline{1} 0)=57013^{\prime} \\
& m: b=(110):(040) \quad 5843 \\
& \mu: c=(1 T 0):(001) \quad 8648 \\
& m: c=(110):(001) \quad 8532 \\
& m: p=(110):(133) \quad 6249
\end{aligned}
$$

Daraus ergibt sich:

$$
\begin{gathered}
a: b: c=0,54737: 1: 0,42196 \\
\alpha=910151^{\prime}, \quad \beta=940181^{\prime}, \quad \gamma=93^{\circ} \mathbf{2 2}^{\prime} .
\end{gathered}
$$

An allen fünf Krystallen, die gemessen wurden, waren die Formen: $c, b, m, \mu, p, \pi$ allgemein verbreitet. Die Form $d$ wurde an vier, die Form $\varepsilon$ an drei Krystallen gefunden, die letzte immer als sehr kleine und schlecht ausgebildete Fläche ${ }^{1}$ ). Ebenfalls sehr schlechte Signale lieferten die Flächen der Formen $f$ und $\varphi$, die an zwei Krystallen sicher gestellt wurden.

Tabelle der gemessenen und berechneten Winkel:

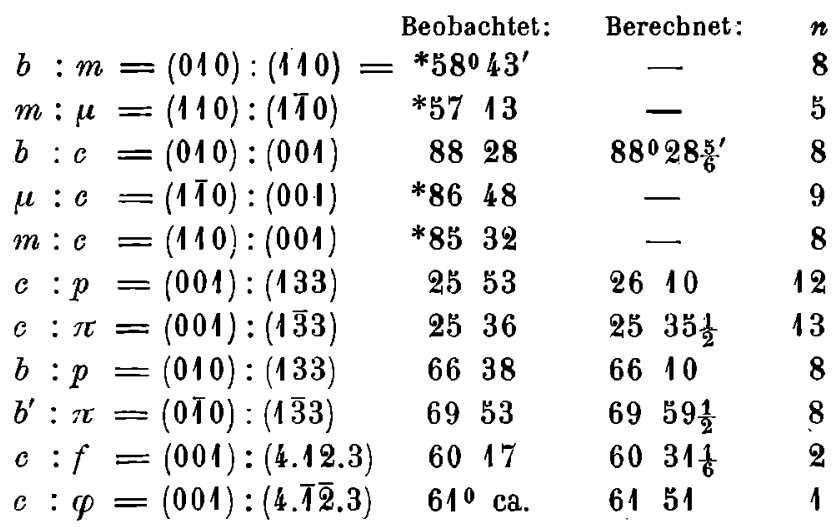

1) Obgleich die Formenindices $d\{\overline{8} 03\}$ und $\varepsilon\{\overline{8} . \overline{2} \overline{4} .3\}$ etwas compliciert erscheinen, ist es unmöglich, sie durch einfachere zu ersetzen; die nächstliegenden einfacheren Indices wären für $d\{\overline{3} 01\}$, für $\varepsilon\{\overline{3} . \overline{9} .1\}$; nun ist

$$
\begin{aligned}
& (\overline{3} 01):(001)=700231^{\prime} \\
& (30 \overline{1}):(110) \quad \mathbf{3 6} 30 \\
& (\overline{3} \overline{9} 1):(001) \quad \mathbf{8 1} 335 \frac{5}{6}
\end{aligned}
$$

was von den in der Tabelle angeführten Werten sehr abweicht. Die Form $\varepsilon$ ist durch die Zonen $[b: d]$ und $[p: c]$ bestimmt, und konnte beim Cadmiumsalze ganz sicher festgestellt werden. 


$\begin{array}{lrrr} & \text { Beobachtet: } & \text { Berechnet: } & n \\ c: d=(001):(\overline{8} 03) & =67054^{\prime} & 67041 \frac{1}{3} & 9 \\ c: \varepsilon=(001):(\overline{8} . \overline{2} \overline{4} .3) & 8011 & 8022 \frac{1}{2} & 2 \\ m: p=(110):(133) & * 6249 & - & 8 \\ \mu: \pi=(1 \overline{1} 0):(1 \overline{3} 3) & 6515 & 6522 \frac{5}{6} & 4 \\ m: d=(110):(80 \overline{3}) & 384 & 3814 & 4 \\ \mu: d=(1 \overline{1} 0):(80 \overline{3}) & 3814 & 3826 \frac{5}{6} & 1 \\ p: \pi=(133):(1 \overline{3} 3) & 4331 & 4350 \frac{1}{2} & 8\end{array}$

Wie oben gesagt wurde, ist die Ausbildung der Krystalle meist dicktafelig nach der Basis. Diese Ausbildungsform von der Combination: c $b m \mu p \pi f \varphi d$ veranschaulicht die Fig. 2. Sie ist durch die prismatische

Fig. 2.

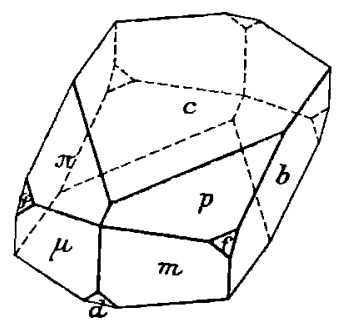

Fig. 3.

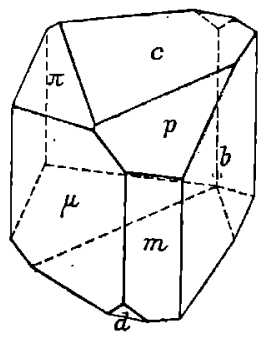

Zone pseudohexagonal, durch die gleichmäBige Ausbildung von $p$ und $\pi$ pseudomonoklin. Ein anderer Typus ist in der Fig. 3 abgebildet; bei diesem ist immer eine der Gegenflächen in der Verticalzone schmal, die andere breit entwickelt, so $\mathrm{da} B$ diese Zone einem trigonalen Prisma ähnlich ist. Als Endflächen treten auf $e\{001\}, p\{133\}, \pi\{1 \overline{3} 3\}$ und $d\{\overline{8} 03\}$. Die Ätzfiguren bewiesen die Gleichwertigkeit der ungleich ausgebildeten Gegenflächen; die asymmetrische Klasse liegt also nicht vor. Den pseudotrigonalen Charakter dieser Krystalle stört die Abwesenheit der Formen $f$ und $\varphi$, welche, wie aus der Projection sichtbar ist, sich mit $d$ zum Rhomboëder ergänzen würden.

Im Konoskope (bei Natriumlicht) zeigen die Krystalle an der Basis links hinten einen Axenaustritt, der $35^{\circ} 44^{\prime}$ gegen die Normale auf $c$ geneigt ist.

Auf der Fläche $\mu(1 \bar{T} 0)$ ist die Axe auch sichtbar, und zwar nur $8^{0} 9^{\prime}$ gegen ihre Normale geneigt.

Auf der Basis beträgt die Auslöschung etwa 70 zur Kante $e: b$ im spitzen Winkel $\gamma$; auf der Fläche $b 40^{\circ}$ gegen die Kante $b: m$ hinauf gegen die Fläche $f$. Auf $m$ ist die Auslöschung $51^{10}{ }^{0}$ von derselben Kante gegen die obere vordere Ecke, und auf $\mu$ etwa $60 \frac{10}{2}$ von der verticalen Kante hinauf gegen die Fläche $\varphi$. 
Einmal wurde Zwillingsbildung beobachtet, bei welcher die Zwillingsebene (001) zugleich als Verwachsungsebene functioniert.

Das spec. Gewicht wurde (in Acetylentetrabromid bei Zimmertempeperatur) zu 1,987 bestimmt.

\section{Äthylendiaminmangansulfat-Tetrahydrat.}

Die Krystalle sind schwach rosa gefärbt, durchsichtig. Ihr Habitus ist immer kurzsäulig nach der Verticalaxe. In der verticalen Zone treten folgende Formen auf: $b\{010\}, m\{110\}, \mu\{1 T 0\}$; die Endigung der Prismen bilden $c\{001\}, \rho\{133\}, \pi\{1 \overline{3} 3\}, f\{4.12 .3\}, \varphi\{4 . \overline{1} \overline{2} .3\}, r\{\overline{2} 63\}, \varrho\{\overline{2} \overline{6} 3\}$, $d\{\overline{8} 03\}$.

Die Flächen der verticalen Zone sind mehr oder minder im Gleichgewichte und demnach die Krystalle einem hexagonalen Prisma ähnlich; oder es dominieren die Prismenflächen gegenüber den Längspinakoiden und bedingen einen säuligen, im Querschnitt rhombischen Typus. Von den Endflächen pflegen die Formen $\pi$ und $\varphi$ stärker ausgebildet zu sein als die analogen Formen $p$ und $f$ der rechten Seite, wodurch das Aussehen unsymmetrisch wird (Fig. 4). Ja es wurden Krystalle gefunden, bei denen die Form $\pi$ größer war als die Basis selbst. Auch in der Prismenzone wurden pseudotrigonale Typen vorgefunden, wie sie beim Eisensalze beschrieben wurden; in der verticalen Zone herrschten drei Flächen der Formen $b, m, \mu$, deren Gegenflächen schmal waren; die Endigung bildeten die Formen $c, p, \pi, r$, $\varrho, d$; letztere zwei Formen sind als sehr schmale Flächen vorhanden. Über den ausgezeichnet pseudotrigonalen Charakter der stereographischen Projection

Fig. 14.

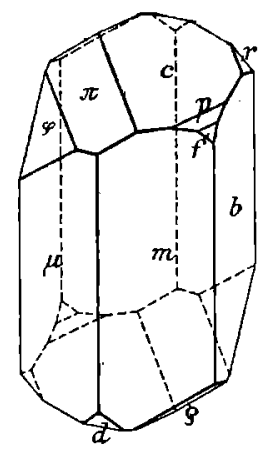
dieses Salzes wurde schon oben gesprochen.

Aus den Winkeln:

berechnet man die Elemente

$$
\begin{aligned}
& m: \mu=(110):(110)=570 \mathrm{I}^{\prime} \\
& \mu: b^{\prime}=(1 \overline{1} 0):(0 \overline{0} 0) \quad 6410 \\
& \mu: c=(1 \overline{1} 0):(001) \quad 8622 \\
& m: c=(110):(001) \quad 8454 \\
& \mu: \pi=(1 \bar{\top} 0):(4 \overline{3} 3) \quad 6454
\end{aligned}
$$

$$
\begin{gathered}
a: b: c=0,54646: 1: 0,42512 \\
\alpha=91^{0} 28 \frac{1}{6}^{\prime}, \beta=94^{0} \quad 53 \frac{1}{2}^{\prime}, \gamma=93^{\circ} \quad 19 \frac{1}{2}^{\prime} .
\end{gathered}
$$

Die Flächen: $b, c, m, \mu, p, \pi$ lieferten in vielen Fällen deutliche bis recht gute Signale; minder gut reflectierten die Flächen $d, f, \varphi$; matt waren und schlechte Signale lieferten die Flächen $r$ und $\varrho$. Gemessen wurden drei Krystalle. 


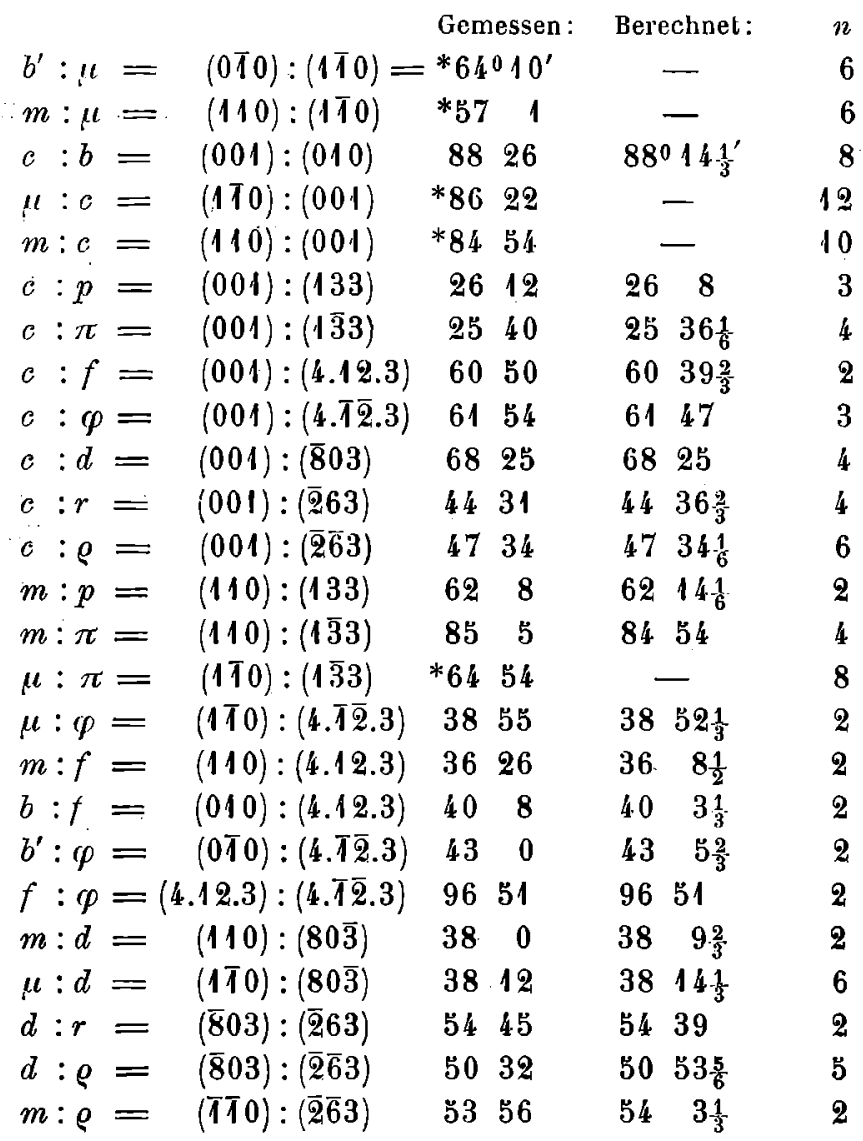

Auf der Fläche $\mu(1 T 0)$ sieht man im Konoskope (bei $N a$-Licht) den Austritt einer Axe links geneigt gegen die Flächennormale um 12016'. Auf der Fläche $b$ löschen die Krystalle unter $40^{\circ}$ von der Kante $b: m$ hinauf gegen $f$ aus, auf $m 47^{\circ}$ von derselben Kante hinauf gegen die vordere obere Ecke, auf $\mu 43 \frac{1}{2}^{0}$ von der verticalen Kante hinauf gegen $\varphi$.

Die Ätzfiguren stimmen gut mit der Symmetrie der pinakoidalen Klasse überein.

Spec. Gewicht (Acetylentetrabromid, Zimmertemperatur) $=1,941$.

\section{Äthylendiamincadmiumsulfat-Tetrahydrat.}

Farblose Krystalle von säulenförmigem Habitus nach der Verticalaxe. Die Flächen der Verticalzone waren an einigen Krystallen etwas gebogen, die Endflächen reflectierten teilweise recht gut. Beobachtet wurden folgende Formen: In der Verticalzone $b\{010\}, m\{1 \mid 0\}, \mu\{1 T 0\}$. Die Endflächen: $e\{001\}, d\{\overline{8} 03\}, p\{133\}, x\{1 \overline{3} 3\}, p\{4 . \bar{T} \overline{2} .3\}, \varrho\{\overline{2} \overline{6} 3\}, r\{\overline{2} 63\}, \varepsilon\{\overline{8} . \overline{2} \overline{4} .3\}$. 
Die Formen $b, m, \mu$ sind entweder im Gleichgewichte entwickelt und erinnern wieder an ein hexagonales Prisma, oder es walten die Prismenflächen vor, die eventuell allein in der Zone vorhanden sind. Die Verteilung der terminalen Formen kann auch bei diesem Salze pseudomonoklines Aussehen bedingen, indem die Basisfläche vorwaltet und die anderen Formen: $p, \pi, r, \varrho, d, \varepsilon$ ajs kleinere Flächen auftreten. Dabei pflegen $p$ und $\pi$ resp. $r$ und $\varrho$ ungefähr von derselben Größe zu sein, die beiden letzteren etwas größer als die ersteren.

Einmal wurde die Kopfausbildung ganz unsymmetrisch gefunden, indem sie von $c, \varrho$ und $\varepsilon$ gebildet wurde.

Diese drei Formen waren ungefähr gleich groß und lieferten gute Signale (Fig. 5). Andere Polausbildung, ebenso unsymmetrisch, bildeten die Formen $c$ und $\pi$, etwa von gleicher Ausdehnung, neben welchen andere

Fig. 5.

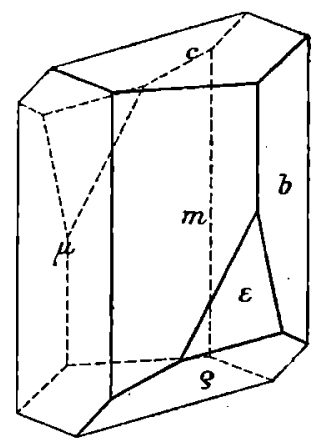

Fig. 6.

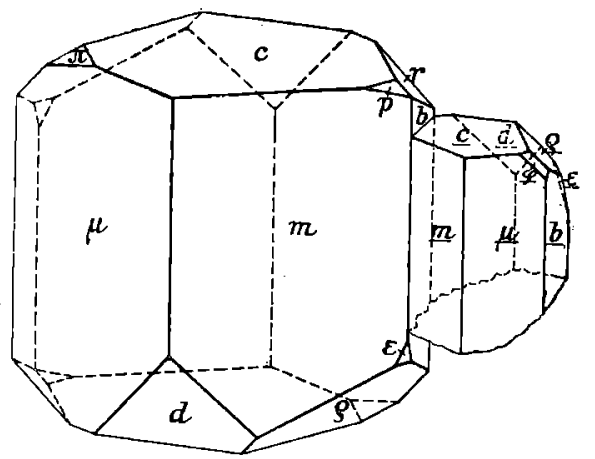

Formen: $p, r, \varrho, \varphi, \varepsilon$ als sehr klein und meistens sehr schlecht reflectierende Flächen vorhanden waren.

Eine Zwillingsbildung wurde nach der Fläche $b$ beobachtet; auf diese Weise verwachsene Individuen (eines derselben viel kleiner als das andere) veranschaulicht die Fig. 6.

Gemessen wurden vier Krystalle. Zum Berechnen der Krystallelemente wurden folgende Winkel benutzt:

$$
\begin{array}{lrr}
m: \mu=(110):(1 \overline{1} 0) & =570 \quad 5^{\prime} \\
m: b=(110):(010) & 58 & 36 \\
c: m=(001):(110) & 85 & 12 \\
c: b=(001):(010) & 88 & 12 \\
c: \varrho=(001):(\overline{2} \overline{6} 3) & 48 & 3
\end{array}
$$

Daraus ergibt sich das Axenverhältnis:

$$
\begin{gathered}
a: b: c=0,54594: 1: 0,43076 ; \\
\alpha=91^{\circ} 302^{\prime}, \beta=94.031 \frac{1}{2}^{\prime}, \gamma=93^{\circ} 32 \underline{z}^{\prime} .
\end{gathered}
$$




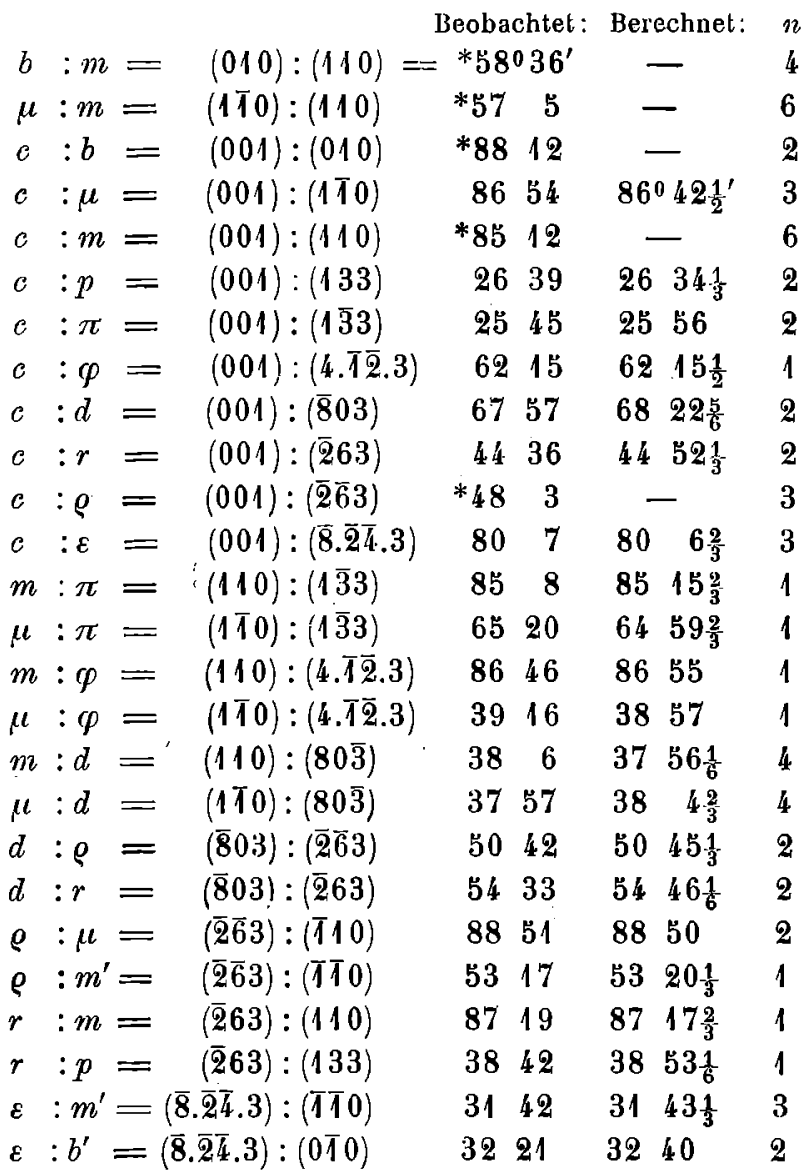

Auf der Fläche $\mu$ sieht man den Austritt der optischen Axe, die links um 12015' gegen die Flächennormale geneigt ist (in $\mathrm{Na}$-Licht). Auf $b$ löschen die Krystalle $40^{\circ}$ gegen die verticale Kante aus, hinauf zur Fläche $p$, auf $m 50^{\circ}$ gegen dieselbe Kante hinauf zur oberen vorderen Ecke, auf $\mu \tau 2 \frac{10}{2}$ gegen die verticale Kante zur Fläche $\varphi$.

Wie bei den vorgehenden Salzen, so stimmen auch bei diesem die Corrosionen mit der pinakoidalen Symmetrie überein.

Spec. Gewicht (Acetylentetrabromid, Zimmertemperatur) $=2,171$.

An den drei untersuchten Salzen wurde also gefunden:

$$
\begin{aligned}
\text { Fe-Salz } \quad a: b: c= & 0,54737: 1: 0,42196 ; \\
& \alpha=91015 \frac{1}{2}^{\prime} ; \beta=94^{0} 181^{\prime} ; \gamma=93^{0} 22^{\prime} . \\
M n- & 0,54646: 1: 0,42512 ; \\
& \alpha=91028 \frac{1}{6}^{\prime} ; \beta=94.053 \frac{1}{2}^{\prime} ; \gamma=93^{\circ} 19 \frac{1}{2}^{\prime} .
\end{aligned}
$$




$$
\begin{aligned}
C d-\text { Salz } \quad a: b: c= & 0,54594: 1: 0,43076 ; \\
& \alpha=91^{0} 300^{2} ; \beta=94031 \frac{1}{2}^{\prime} ; \gamma=93^{\circ} 32 y^{\prime} 5^{\prime} .
\end{aligned}
$$

Aus dieser Zusammenstellung ist die nahe Übereinstimmung der Krystallelemente der drei Salze zu ersehen; bei den Parameterverbältnissen $a: b$ befindet sich eine Differenz erst in der vierten Decimale, bei $a: b$ in der dritten Decimale.

In den Werten $a: b$ und $c: b$ herrscht eine auffallende Regelmäßigkeit; vom Eisensalze zum Mangansalze und Cadmiumsalze sinkt der Wert $a: b$, während $c: b$ in derselben Richtung rascher steigt. Die Werte des Mangansalzes sind also in der Mitte zwischen denen des Eisen- und Cadmiumsalzes.

Berechnen wir aus den Molekulargewichten, spec. Gewichten und Krystallelementen die topischen Parameter, so bekommen wir folgende Zahlen:

$$
\begin{aligned}
& \text { M.-Gew.: Sp. Gew.: } \quad \text { Äq.-V.: } \quad \chi \quad \psi \quad \psi \quad \omega \\
& \text { Fe-Salz } \quad 382,18 ; \quad 1,987 ; \quad 192,34 ; \quad 5,158: 9,423: 3,976 \\
& M n-\quad 381,28 ; \quad 1,941 ; 196,49 ; \quad 5,178: 9,475: 4,028 \\
& C d-438,68 ; 2,171 ; 202,06 ; 5,199: 9,524: 4,103 \text {. }
\end{aligned}
$$

Das Mangansalz steht also mit seinem Äquivalentvolumen und seinen topischen Axen in der Mitte zwischen dem Eisen- und dem Cadmiumsalze. Alle diese Größen wachsen in der Richtung von Eisen- zum Mangan- und Cadmiumsalze, und zwar die Differenzen $\chi_{H n}-\chi_{F e}$ resp. $\psi_{M n}-\psi_{F e}$ sind ungefähr gleich denen: $\chi_{C d}-\chi_{11 n}$ resp. $\psi_{C d}-\psi_{\mathbb{M} n}$. Nur die Differenz $\omega_{C d}-\omega_{M n}$ ist etwas größer als diejenige $\omega_{M n n}-\omega_{F^{\prime} e}$.

Diese Regelmäßigkeit entspricht aber weder den spec. noch den Molekulargewichten, welche beide Werte beim Mangansalze niedriger sind als beim Eisensalze. Es liegt also ein Fall vor, in welchem die krystallographischen Eigenschaften nicht die Functionen der Atomgewichte der einander substituierenden Elemente sind ${ }^{\text {) }}$.

Um zu prüfen, ob ähnliche RegelmäBigkeiten sich auch aus anderen Beispielen ableiten lassen, benutzte ich zum Vergleiche drei Reihen analoger Eisen- resp. Mangan- und Cadmiumsalze:
1) $\mathrm{Rb}_{2} \mathrm{M}\left(\mathrm{SO}_{4}\right)_{2} .6$ aq, wo $\mathrm{M}=\mathrm{Fe}, \mathrm{Mn}, \mathrm{Cd}$;
2) $\mathrm{Cs}_{2} \mathrm{M}\left(\mathrm{SO}_{4}\right)_{2} .6 \mathrm{aq}, \quad-\mathrm{M}=\mathrm{Fe}, \mathrm{Mn}, \mathrm{Cd}$;
3) $\mathrm{K}_{2} M\left(\mathrm{SO}_{4}\right)_{2} .2 \mathrm{aq}, \quad-M=\mathrm{Fe}, \mathrm{Mn}, \mathrm{Cd}$.

Für die ersten zwei Reihen wurden die topischen Axen von Tutton ${ }^{2}$ )

1) Dieses Resultat ist also ganz analog demjenigen, welches $T u t$ ton für Thallium im Vergleiche mit den Alkalimetallen fand (diese Zeitschr. 44, 113); es ist aber auffallend, daß die Substitution von $F e$ durch $C d$ regelmäßig die Krystallelemente ändert, während das dem Eisen im periodischen Systeme viel näher stehende $M n$ eine Ausnahme von der Regel macht.

2) Diese Zeitschr. 27, 113. 
angegeben, die dritte Reihe untersuchte Wyrouboff 1 und aus dessen Werten berechnete jch die topischen Axen.

\begin{tabular}{|c|c|c|c|c|}
\hline Ad 1) & M.-Gew.: & Sp. Gew.: & Äquiv.-Vol.: & $\psi$ \\
\hline $\mathrm{Rb}_{2} \mathrm{Fe}\left(\mathrm{SO}_{4}\right)_{2} .6 \mathrm{aq}:$ & 526,4 & 2,516 & 209,22 & $6,1832: 8,3817: 4,1942$ \\
\hline $\mathrm{Rb}_{2} \mathrm{Mn}\left(\mathrm{SO}_{4}\right)_{2} \cdot 6 \mathrm{aq}:$ & 525,4 & 2,459 & 213,66 & $6,2542: 8,4723: 4,1938$ \\
\hline$R b_{2} C d\left(S O_{4}\right)_{2} .6 \mathrm{aq}:$ & 582,4 & 2,672 & 217,96 & $6,2828: 8,5526: 4,2174$ \\
\hline
\end{tabular}

Ad 2)

$\begin{array}{rrrrr}\mathrm{Cs} \mathrm{se}_{2}\left(\mathrm{SO}_{4}\right)_{2} .6 \mathrm{aq}: & 621,4 & 2,791 & 222,64 & 6,2806: 8,6497: 4,2842 \\ \mathrm{Cs}_{2} \mathrm{Mn}\left(\mathrm{SO}_{4}\right)_{2} .6 \mathrm{aq}: & 620,4 & \mathbf{2 , 7 3 8} & \mathbf{2 2 6 , 5 9} & \mathbf{6 , 3 4 4 1 : 8 , 7 2 1 7 : 4 , 2 8 5 0} \\ \mathrm{Cs}_{2} \mathrm{Cd}\left(\mathrm{SO}_{4}\right)_{2} .6 \mathrm{aq}: & 677,4 & 2,955 & 229,24 & 6,3638: 8,7668: 4,3010\end{array}$

Bei der zweiten Reihe sind die Verhältnisse offenbar genau dieselben, wie es bei den untersuchten Äthylendiaminsulfaten der Fall war: während das Molekular- und Volumgewicht des Mangansalzes ān der Spitze der Reihe stehen, sind die Werte von Äquiv.-Volumen und von $\%, \psi, \omega$ zwischen denselben Werten von Eisen und Cadmium. Die erwähnten Werte steigen in der Richtung vom Eisen zum Mangan und Cadmium. Bei der Reihe der Rubidiumdoppelsulfate macht eine einzige Ausnahme von der Regel der Wert $\omega$, welcher bei dem $M n$-Salze etwas kleiner ist als beim Eisensalze. Weil aber der Wert $\omega$ überhaupt unbedeutende Veränderung erleidet, hat diese Ausnahme keine größere Störung der Regel zur Folge.

Diesen übereinstimmenden Resultaten gegenüber stehen die Verhältnisse bei den von Wyrouboff untersuchten Dihydraten:

\begin{tabular}{|c|c|c|c|c|}
\hline & M.-Gew.: & Sp. Gew.: & Äquiv.-Vol.: & $\omega$ \\
\hline $\mathrm{FeK}_{2}\left(\mathrm{SO}_{4}\right)_{2}$ & 362,35 & 2,683 & 135,05 & $5,391: 7,578: 3,411$ \\
\hline $\mathrm{MnK}_{2}\left(\mathrm{SO}_{4}\right)_{2} \cdot 2 \mathrm{aq}:$ & $361,4.5$ & 2,632 & 137,33 & $5,445: 7,603: 3,408$ \\
\hline$C d K_{2}\left(S_{4}\right)_{2} \cdot 2 \mathrm{aq}:$ & 418,85 & 2,922 & 143,34 & $\left.6,104: 7,661: 3,250^{2}\right)$ \\
\hline
\end{tabular}

Bei dieser Reihe sind die Verhältnisse in Betreff des Molekular- und spec. Gewichtes, des Äquiv.-Volums und der topischen Parameter $\chi$ und $\psi$ ganz ähnliche wie bei den oben angeführten Beispielen; die letztgenannten Werte des Mangansalzes stehen wieder zwischen denselben Werten von Eisen- und Cadmiurnsalz, wobei die Differenzen $\chi_{c d}-\chi_{M n n}, \psi_{c d}-\psi_{M n}, \ddot{A} .-\mathrm{V} . c_{d}-\ddot{A} .-\mathrm{V} \cdot \mathbb{M n}$ beträchtlich größer sind als die entsprechenden Differenzen $\chi_{\text {Inn }}-\chi_{F_{e}}$, $\psi_{M_{n}}-\psi_{F^{\prime} \ell}, \ddot{\mathrm{A}} .-V_{\mu_{n}}-\ddot{A} .-V_{F_{\ell}}$. Alle die genannten Werte steigen in der Richtung vom Eisensalze zum Mangan- und Cadmiumsalze. Der Wert $\omega$ aber verhält sich umgekehrt, indem er in derselben Richtung sinkt.

1) Bull. de la soc. min. d. la Fr. 14, 233 . Ref. diese Zeitschr. 22, 191.

2) Wyrouboff gibt für diese Krystalle zwei Stellungen an; die angefübrten Zahlen beziehen sich auf seine erste Position; für die zweite wären dieselben:

$$
\varkappa=5,888, \quad \psi=7,660, \quad \omega=3,249 .
$$


Die Differenz $\omega_{M M_{h}}-\omega_{C_{d}}$ ist wieder größer als diejenige $\omega_{H_{e}}-\omega_{M Y_{n}}$. Am wenigsten indert sich der Wert $\psi$ aller drei Verbindungen.

Es wird sicher von großem Interesse sein, an mehreren isomorphen Salzen der Elemente $\mathrm{Fe}, \mathrm{Mn}, \mathrm{Cd}$ die morphotropen Wirkungen zu prüfen.

Ein Resultat jedoch kann man aus den vorliegenden Untersuchungen ableiten, nämlich, daß in der Reihe der Eisen-Mangan-Cadmium-Salze Mangan eine besondere Rolle spielt, indem es mit seinem Molekulargewichte und specifischem Gewichte auf der einen Seite, und mit dem ÄquivalentVolumen und topischen Parametern an der anderen nicht in die Reihe paßt.

\section{Monoäthylenxanthonsäure $+H_{2}$.}

Schmelzp. 1250. Krystalle aus dem chem. Staatslaboratorium München.

Durchsichtige, citrongelbe Krystalle, meist von tafeligem Habitus nach der Basis $c\{001\}$. Krystallsystem: rhombisch bipyramidal.

Beobachtete Formen (an drei gemessenen Krystallen): $b\{010\}, c\{001\}$, $n\{120\}, k\{012\}, o\{111\}, i\{121\}$. Die Flächen der Form $c$ sind meistens vorwaltend und auch recht gut glänzend, seltener matt oder durch eine Einsenkung gestört. Auf einigen Krystallen aber erreichen auch die $b$-Flächen bedeutendere Größe und bedingen einen dicktafeligen Habitus. Das Brachydoma $k$ war immer vorhanden, und es wurden auch Fälle beobachtet, wo diese Form größer war als die Basis, doch die Reflexe ihrer Flächen waren immer verwischt oder kettenförmig ausgedehnt. Von allgemeiner Verbreitung sind auch die Formen $b, o$ und $i$, deren Flächen manchmal gute Signale lie-

Fig. 7 .

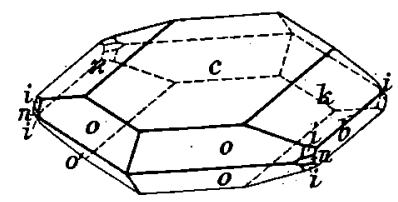
ferten; die Form $i$ pflegt oft nur mit einigen Flächen ausgebildet zu sein Seltener ist das Prisma in, dessen Flächen immer schlechte Signale darboten.

Den gewöhnlichen Typus stellt die Fig. 7 dar (etwas stärker nach vorne gedreht).

Aus den Winkeln: $\quad c: 0=54012^{\prime}$

$$
b: o=6529
$$

wurde das Axenverhältnis berechnet:

$\begin{array}{lccr}0,59546: 1: 0,70937 . \\ & \text { Gemessen: } & \text { Berechnet: } & n \\ n: b=(120):(010)=40^{0} 40^{\prime} & 40^{0} 1 \frac{1 !}{6} & 2 \\ k: c=(012):(001) & 1941 & 1931 \frac{2}{3} & 22 \\ o: b=(111):(010) & 6529 & - & 14 \\ o: c=(111):(001) & 5412 & - & 24 \\ i: b=(121):(010) & 4758 & 4737 \frac{5}{6} & 6 \\ i: c=(121):(001) & 6144 & 6138 \frac{1}{3} & 3\end{array}$


Spec. Gewicht $=1,255$ (in Thouletscher Lösung). Die Ebene der optischen Axen $=(100)$. Auf der Basis tritt die stumpfe, auf $b$ die spitze Bisectrix aus. Der Axenwinkel wurde in der Thouletschen Lösung $(n=$ 1,7252) gemessen (in $\mathrm{Na}$-Licht). Dabei wurde gefunden:

Auf der Fläche $c$ 2 $H_{0}=93015^{\prime}$

$$
\text { - } \quad \text { - } b 2 I I_{a}=7354
$$

Daraus der wahre Axenwinkel

$$
2 V_{a}=79011^{\prime}, \beta=1,627 \text {. }
$$

\section{Hydrochinontetracarbonsäure-Dimethyläther-}

Tetramethylester. Schmelzp. $95^{0}$.

$$
\begin{gathered}
\mathrm{COCH}_{3} \\
\mathrm{H}_{5} \mathrm{C}_{2} \cdot \mathrm{O}_{2} \mathrm{CC}^{\mathrm{C}} \mathrm{CCO}_{2} \mathrm{C}_{2} \mathrm{H}_{5} \\
\mathrm{H}_{5} \mathrm{C}_{2} . \mathrm{O}_{2} \mathrm{C} C \quad \mathrm{ICCO}_{2} \cdot \mathrm{C}_{2} \mathrm{H}_{5} \\
\mathrm{COCH}_{3}^{C}
\end{gathered} .
$$

Dargestellt von Nef (Amer. chem. Journ. 1889, 11, 12).

Die Krystalle sind von paraffinähnlichem. Ansehen, weiß und wenig durchscheinend; ihr Habitus ist säulen- bis tafelförmig. Krystallsystem: Monoklin. Beobachtet wurden folgende Formen: $c\{001\}, b\{010\}, m\{110\}$, $\omega\{(111\}, q\{421\}, x\{(\overline{0} 0.10 .1)(?)$. Gemessen wurden fünf Krystalle.

Die säulenförmig ausgebildeten Krystalle haben die Formen $b$ und $m$ fast im Gleichgewichte und gehen in tafelförmige über, indem entweder

Fig. 8.

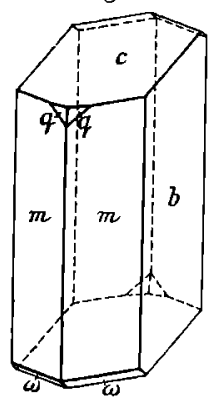

Fig. 9.

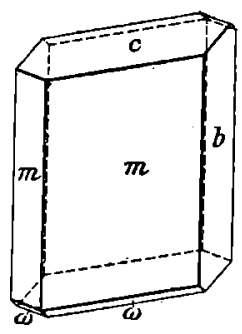
die Flächen $b$ oder ein Paar der Flächen $m$ vorwalten. Einige der Flächen $m, b$ und $c$ reflectierten ziemlich gut, andere dagegen waren ganz matt oder krumm. Immer schlecht reflectierten die Flächen der Form $\omega$, die nicht allgemein vorhanden waren, sowie die Formen $q$ und $\pi$, die beide nur einmal beobachtet wurden. Die letzte Form betrachte ich als nicht ganz sicher. Die Fig. 8 veranschaulicht einen nach der Fläche $b$ dicktafeligen Krystall von der Combination: $b, m, c, \omega, q$. In der Fig. 9 ist ein nach den $m$-Flächen tafeliger Krystall abgebildet.

Aus den Winkeln:

$$
\begin{aligned}
& m: m=(110):(1 \overline{1} 0)=63^{0} 27^{\prime} \quad \text { 21 } \\
& m: c=(110):(001) \quad 77 \quad 4 \quad 28 \\
& \omega: o=(\overline{1} 11):(001) \quad 40 \quad 1 \quad 4 \text {, }
\end{aligned}
$$


wurde berechnet: $\quad a: b: c=0,64084: 1: 0,39365 ; \beta=105015$.

\begin{tabular}{|c|c|c|c|c|}
\hline$\omega$ & $: b=$ & $(\overline{1} 11):(010)=$ & $\begin{array}{l}\text { Gemessen: } \\
68^{0} 55^{\prime}\end{array}$ & $\begin{array}{c}\text { Berechnet: } \\
69^{\circ} 42^{\prime}\end{array}$ \\
\hline$q$ & $: c=$ & $(421):(001)$ & 5514 & 5631 \\
\hline$q$ & $: m=$ & $(421):(110)$ & 4939 & 506 \\
\hline & $: c=$ & $.10 .1):(001)$ & 9454 & 95 \\
\hline
\end{tabular}

Spec. Gewicht (Thouletsche Lösung) $=1,276$.

Die Ebene der optischen Axen ist senkrecht zum Längspinakoid, ca. $39^{\circ}$ von der Kante $b: m$ in den stumpfen Winkel $\beta$ geneigt. Auf der Fläche $m$ beträgt die Auslöschungsschiefe $38^{0}$ von der Kante $b: m$.

\section{Dioxytriphenylmethancarbonsäure}

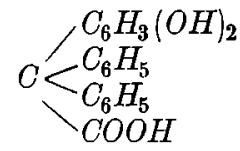

Krystalle aus dem Laboratorium für angewandte Chemie in München.

Die Krystalle sind von blaßgelber Farbe, monoklin. Die beobachteten Formen sind: $a\{100\}, b\{010\}, c\{001\}, m\{110\}, p\{\overline{2} 11\}$; außerdem wurden noch mehrere Formen beobachtet, die aber eher Kanten ähnlich waren und meistens sehr ungenau auf Schimmer gemessen werden konnten. Es sind: $\{310\},\{113\},\{\overline{6} 23\},\{\overline{6} 34\}$. Gemessen wurden vier Krystalle.

Die Formen $c$ und $m$ herrschen immer vor und bedingen einen tafeligen bis kurzprismatischen Habitus. $c$ und $m$ reflectierten teils gut, teils - bei rauher Oberfläche - lieferten sie schlechte Signale. Doch auch bei den schärfsten Signalen konnte die Ablesung nicht immer genau vorgenommen werden, weil die Flächen entweder anomal gelagert oder durch Vicinalen ersetzt waren. Aus diesen störenden Umständen erklärt sich die manchmal wenig genaue Übereinstimmung der gemessenen und berechneten Werte.

$p$ wurde auf allen Krystallen gefunden oft als matte, seltener als glatte und ziemlich gut reflectierende Fläche. $a$ und $b$ waren immer als sehr schmale und schlecht ausgebildete Flächen vorhanden. Ihre Verbreitung ist nicht allgemein.

Fig. 10 ist für die Combination $e, m, p, a$, $b$ gezeichnet.

Aus den Winkeln:

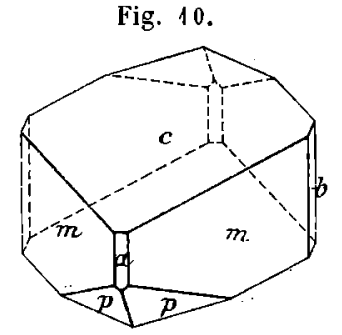

$$
\begin{aligned}
& (110):(\overline{1} 10)=92018^{\prime} \\
& (001):(110) \quad 6834 \\
& (\overline{2} 11):(\overline{2} \overline{1} 1) \quad 57 \quad 13
\end{aligned}
$$

wurden die Elemente berechnet: 


$$
\begin{aligned}
& a: b: c=1,11430: 1: 0,81194 ; \beta=120^{0} 262^{\prime} . \\
& \text { Beobachtet: Berechnet: } n \\
& m: m=(110):(110)=* 92018^{\prime} \quad-16 \\
& m: c=(110):(001) \quad * 6834 \quad-\quad 32 \\
& m: p=\left(T_{10}\right):(\overline{2} 11) \quad 3718 \quad 36^{0} 48 \frac{1}{6} \quad 14 \\
& p: c=(\overline{2} 11):(001) \quad 79 \quad 2 \quad 7941 \frac{1}{3} \quad 22 \\
& p: p=(\overline{2} 11):(\overline{2} \overline{1} 1) \quad * 5713 \quad-\quad 12
\end{aligned}
$$

\begin{tabular}{|c|c|}
\hline$(110):(310)=26^{0} \mathrm{5}^{\prime}$ & $26^{0} \quad 5^{\prime}$ \\
\hline$(110):(113)=5351$ & $5258 \frac{1}{3}$ \\
\hline$(\overline{2} 11):(\overline{6} 23)=10 \quad 40$ & $837 \frac{2}{3}$ \\
\hline$(\overline{2} 11):(\overline{6} 34)=1124$ & 12 \\
\hline
\end{tabular}

Auf den kantenähnlichen Formen wurde gemessen:

Spec. Gewicht (Thoulet sche Lösung) $=1$,293. Keine Spaltbarkeit wurde beobachtet.

Auf der Basis tritt die stumpfe Bisectrix $c$ aus, die etwas nach hinten von der Flächennormale zum negativen Pole der Axe a geneigt ist. Die Ebene der optischen Axen ist senkrecht zu $b$ orientiert.

Starke Licht- und Doppelbrechung. Mit dem Pulfrich-Abbeschen Totalrefractometer wurden die Brechungsexponenten gemessen (auf der Basis, nach Violas Methode):

$$
\begin{aligned}
& \alpha=1,5973, \quad \gamma-\alpha=0,1006, \\
& \beta=1,6587, \quad \beta-\alpha=0,0614, \\
& \gamma=1,6979, \quad \gamma-\beta=0,0392,
\end{aligned}
$$

$\beta$ ist näher an $\gamma$ als an $\alpha$, die Substanz ist also optisch negativ.

Aus einem der Krystalle wurde ein Schliff \| der längspinakoidalen Fläche $b$ hergestellt; auf demselben konnte gemessen werden: die Auslöschung zu etwa 70 schief von der Kante (110):(1T0) im stumpfen Winkel $\beta$ (Richtung der Mittellinie a); der scheinbare Axenwinkel in Natriumlicht mit dem Axenwinkelapparate gemessen in Thouletscher Lösung

$$
\begin{aligned}
& 2 H_{a}=74010^{\prime} . \\
& 2 V_{a}=77018^{\prime} \\
& 2 V_{o}=10242 .
\end{aligned}
$$

Daraus

Die spitze, parallel der $b$-Axe laufende Bisetrix ist $\mathfrak{a}$.

Berechnen wir aus den Brechungsexponenten den wahren Axenwinkel, so bekommen wir

$$
2 V=105018 \frac{2}{3}
$$

was dem gemessenen Werte recht nahe steht. 


\section{Cuminyltoluidin}

$\mathrm{C}_{3} \mathrm{H}_{7} \cdot \mathrm{C}_{6} \mathrm{H}_{4} \cdot \mathrm{CH}_{2} \cdot \mathrm{NH} \cdot \mathrm{C}_{6} \mathrm{H}_{4} \cdot \mathrm{CH}_{3}$. Schmelzp. $36^{0}$. Krystalle aus Äther.

Dargestellt von Übel (Ann. d. Chemie 245, 293).

Die Krystalle sind von langsäuligem Habitus und hellbrauner Farbe. Krystallsystem: Triklin. Die Combinationen sind sehr einfach: $c\{001\}$, $a\{100\}, b\{010\}, \varrho\{\overline{1} 01\}, x\{0 \overline{1} 1\}, \tau\{0 \bar{T} 2\}$.

Die Prismen sind nach der Axe $b$ gestreckt. Die Pinakoide $c\{001\}$ und $a\{100\}$ pflegen etwa im Gleichgewichte zu sein. Die Form $\varrho$ wurde auf einem Krystalle als vereinzelte schmale Facette gefunden. Das Brachydoma $x\{0 T 1\}$ wurde an fünf Krystallen sichergestellt als schmale, die Kante $e: b$ abstumpfende Flächen. Gute Reflexe lieferte es nur auf einem Krystalle. Einmal wurde die Form $\tau$ gefunden als matte sehr schmale Facette, die mit vorgeschlagener Lupe auf Schimmer gemessen werden konnte. Recht gute Signale bot die Form $b$. Insgesamt wurden 13 Krystalle gemessen. Den Typus der Krystalle veranschaulicht die Fig. 11.

Fig. 11.

Zur Berechnung der Krystallelemente wurden folgende Winkel benutzt:

$$
\begin{array}{lrr}
c: a=(001):(100)=68059^{\prime} & 52 \\
c: Q=(001):(101) & 4219 & 1 \\
a: b=(100):(010) & \mathbf{8 8} 40 & 12 \\
c: b=(001):(010) & 5938 & 8 \\
a: x=(100):(011) & \mathbf{7 2} 34 & 6
\end{array}
$$

Daraus ergibt sich:

$$
\begin{gathered}
a: b: c=1,5279: 1: 1,2793 ; \\
a=122011 \frac{2}{3}^{\prime}, \beta=113^{0} 422^{\prime}, \gamma=780411^{\prime} .
\end{gathered}
$$

Für die übrigen Winkel wurde gefunden:

$$
\begin{aligned}
& \text { Beobachtet: Berechnet: } n \\
& c: x=(001):(0 \pi 1)=68^{0} 51^{\prime} \quad 68^{0} 58^{2} \quad 10 \\
& c: \tau=(001):(0 \overline{1} 2) \quad 36 \quad 3 \quad 36261
\end{aligned}
$$

Die Spaltbarkeit ist vollkommen nach a (100), deutlich nach $c(001)$.

Auf der Basis ist die Auslöschung fast parallel der Kante $c: b$, auf dem Querpinakoide $a$ etwa $17^{\circ}$ von der Kante $a: c$ im stumpfen Winkel $\alpha$; im monochromatischen, convergenten ( $\mathrm{Na}$-)Lichte sieht man auf den Spaltflächen nach $a$ eine Axe schief austreten.

Spec. Gewicht $=1,108$. 


\section{Das normale Ammoniumorthosulforanadat $\left[\mathrm{NH}_{4}\right]_{3} \mathrm{VS}_{4}$.}

Dargestellt von Schultze, Dissertation München 1908.

Nadelige, stark metallglänzende Krystalle, auch in dünnsten Splittern undurchsichtig. Daneben finden sich Übergänge zu gekrümmten, oft skelettartig entwickelten Gebilden. Endflächen sind nur an einem Pole vorhanden.

Krystallsystem: Monoklin. Die Krystalle zeigen folgende Combination: $a\{100\}, m\{110\}, o\{111\}$. Man kann zwei Typen unterscheiden: 1) Alle

Fig. 12.

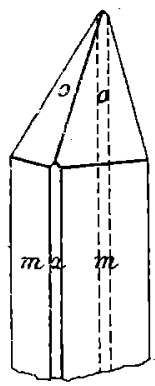

Fig. 13.

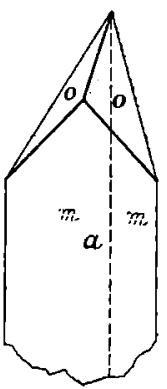
vier Prismenflächen sind mehr oder minder im Gleichgewichte, $a$ ist schmal; als Endflächen sind Pyramidenflächen ausgebildet. Fig. 12.

2) Das Prisma ist nur mit zwei Flächen vorhanden, $\{\overline{1} \overline{0} 0\}$ und $\{\overline{1} 10\}$, das Pinakoid $a$ nur mit einer breiten Fläche $\{100\}$. Am Pole wieder die Pyramide. Fig. 13.

Gute Signale lieferten einige Flächen des Pinakoids und des Prismas. Die Pyramidenflächen reflectierten minder gut, zum Teil sogar sehr schlecht. Wegen. der Kleinheit der Krystalle mußte man in vielen Fällen die Messungen mit verkleinerndem Ocular durchführen.

An zehn Krystallen wurde gefunden:

Gemessen :

$$
\begin{aligned}
& a: m=(100):(110)=* 49019^{\prime} \\
& m: o=(\overline{1} 10):(111) \\
& \text { *83 } 17 \\
& o: o=(111):(1 T 1) \\
& * 74 \quad 39 \\
& m: o=(110):(111) \\
& 36 \quad 15
\end{aligned}
$$$$
\text { Berechnet: } \quad n
$$$$
\text { - } 35
$$

\begin{tabular}{|c|c|c|c|}
\hline 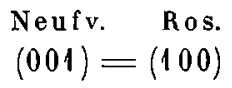 & $\begin{array}{l}\left.\text { eufv. ber. }{ }^{1}\right) \\
(1):(1 / 1)\end{array}$ & $\begin{array}{l}\text { rhomb.) } \\
\text { '4059' }\end{array}$ & $\begin{array}{l}\text { Ros. ber. als mono } \\
(111):(1 \overline{1} \mid)=74^{0}\end{array}$ \\
\hline$(101) \quad(11$ & $(111) \quad(001)$ & 5830 & $(111)(100) \quad 5$ \\
\hline
\end{tabular}

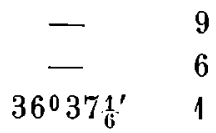

Daraus ergibt sich das Axenverhältnis:

$$
1,1634: 1: 1,0165 ; \beta=90^{\circ} 27^{\prime} \text {. }
$$

Nach De Neufville (Liebigs Annalen der Chemie, 263, 48; diese Zeitschr. 23, 318) ist diese Substanz rhombisch; dabei wählte er eine andere Position, bei welcher $(h k l)=(l h k)$ nach meiner Position:

1) Die letzten zwei Zahlen der Colonne seufv. ber. wurden von mir nach Neufvilles Grundwinkeln berechnet. Im Originale von Neufvilles Messungen sind Druckfehler, indem die Pyramidenflächen immer gleich bezeichnet sind. Diese Fehler wurden im Ref. Muthmanns beseitigt. 


\begin{tabular}{|c|c|c|c|c|c|c|c|c|}
\hline $\begin{array}{l}\text { Neufv.: Ros.: } \\
(111)=(111)\end{array}$ & $\begin{array}{l}\text { Neuf } \\
(111)\end{array}$ & $\begin{array}{l}\text { ber. } \\
:(101)\end{array}$ & $3 t$ & 4.) : & $\begin{array}{l}\text { Ros. } \\
(1+1)\end{array}$ & $\begin{array}{l}\text { ber. } \\
(1 \mathbf{1} 10)\end{array}$ & $\begin{array}{r}\text { honok } \\
36^{\circ}\end{array}$ & $\begin{array}{l}3 .): \\
37 \frac{1}{6}\end{array}$ \\
\hline$(\bar{T} 11) \quad(1 \bar{T} 1)$ & $(110)$ & $(1 \bar{T} 0)$ & & 2 & $(0+1)$ & $(01 \pi)$ & 89 & $3 \frac{2}{3}$ \\
\hline$(110)$ & $\left(\begin{array}{lll}1 & 0 & 1\end{array}\right)$ & $(001)$ & 49 & 25 & $(110)$ & $(100)$ & 49 & 19 \\
\hline & $(10 \bar{T})$ & $(111)$ & 82 & $55 \frac{5}{6}$ & $(T 10)$ & $(111)$ & 83 & 17 \\
\hline
\end{tabular}

Nach Neufville sind die Krystalle manchmal nach der Axe $c$ gestreckt (auch an den Figuren), das ist nach $\{011\}$ in meiner Position. Weder solche Ausbildung, noch diese Form überhaupt konnte. ich beobachten. Desgleichen konnte ich niemals die Form $\{$ T11\} sicher stellen, welche sich mit $\{111\}$ zur rhombischen Pyramide ergänzen könnte. Von den Pyramidenflächen sagt Neufville, daß sie ungleich groß entwickelt sind.

Dabei ist nötig, einige irrtümliche Angaben Neufvilles zu corrigieren Aus den. Grundwinkeln Neufvilles:

$$
\begin{aligned}
& (111):(1 T 1)=* 73028^{\prime} \\
& (111):(\overline{1} 11) \quad * 7459
\end{aligned}
$$

folgt das Axenverhältnis: 0,9826:1: 1,1471 , während Neufville angibt: $0,9825: 1: 1,1741$ (Druckfebler?).

Der Wert (001):(111) soll heißen 58034'30" statt $58^{\circ} 30^{\prime}$ Neufvilles (diese Differenz ändert die Verticalaxe schon um vier Einheiten der dritten Decimale).

Der Wert $(110):(1 \bar{T} 0)$ soll heißen $88^{\circ} 59^{\circ} \frac{2}{3}^{\prime}$ statt $89^{\circ} 2^{\prime}$.

Aus Neufvilles Axenverhältnisse resultiert weiter: (101):(001)= כ0 $0^{0} 4 \frac{5^{\prime}}{6}$, während derselbe Flächenwinkel, aus dem richtigen Axenverhältnisse berechnet, heißen soll: $49^{\circ} 25$.

Die Mischkrystalle mit etwa $5 \% K$ besitzen das Axenverhältnis nach Neufville (l. c. 59):0,9873:1:1,4294; diese Größen wollte Neufville aus den Werten:

$$
[170]:[110]=89^{\circ 15^{\prime}},[111]:[110]=23^{\circ} 45^{\prime}
$$

berechnet haben. De facto resultiert aus seinen Winkeln das Axenverhältnis: 0,9870:1:1,5965. Aus Neufvilles Parametern ergeben sich die zugrunde gelegten Winkelgrößen wie folgt:

$$
\begin{aligned}
& {[1 \overline{1} 0]:[110]=89^{016^{\prime}}} \\
& {[110]:[111] \quad 26010^{\prime} 30^{\prime \prime} .}
\end{aligned}
$$

Der Rechnungsfehler macht sich also im zweiten Winkel sehr erkennbar. 


\section{Athyleudiaminkupfersulfat-Hexahydrat$$
\mathrm{Cu}\left[\mathrm{SO}_{4}\right]_{2} \underset{\mathrm{CH}}{\stackrel{\mathrm{CH}}{\mathrm{C}} \cdot \mathrm{NH}_{2} \cdot \mathrm{NH}_{2}}+6 \text { aq. }
$$

Die Krystalle sind von tiefblauer Farbe und kurzsäuligem Habitus. Krystallsystem: Monoklin. Die gefundene Combination ist: $m\{110\}, q\{011\}$,

Fig. 14.

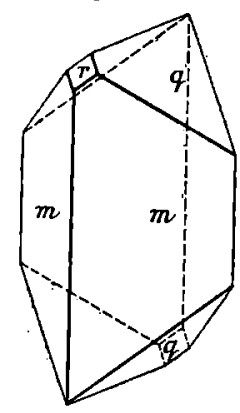
$r\{101\}$.

Meistens herrschen die Prismenflächen, die oft gewölbt und uneben sind, vor; bei anderen Krystallen aber waren die Prismen- und Klinodomenflächen etwa im Gleichgewichte; oder endlich waren ein Paar der Klinodomenflächen recht groß ausgebildet und verliehen den Krystallen einen asymmetrischen Gharakter. Fig. 14 stellt einen nach den Prismenflächen kurzsäuligen Typus vor mit den $q$-Flächen normal ausgebildet und mit dem Orthodoma $r$, welches auf zwei Individuen als kleine, schlecht reflectierende Fläche gefunden wurde. Insgesamt wurden sieben Krystalle untersucht. Den pseudorhombischen Charakter hatte Dr. H. Steinmetzi) erwähnt.

Aus den Wink

$$
\begin{array}{lrl}
q: q^{\prime}=(011):(0 \pi 1)= & 82^{0} 24^{\prime} & n \\
m: q=(110):(011) & 6130 & 18 \\
m: q^{\prime}=(110):(0 \pi 1) & 11229 & 15
\end{array}
$$

folgt: $\quad a: b: c=0,86397: 1: 0,87847 ; \beta=94046^{\prime}$.

Die übrigen Winkelgrößen sind:

$$
\begin{aligned}
& m: m^{\prime}=(110):(1 \overline{1} 0)=81^{0} 30^{\prime} \text { beob., } \quad 810271^{\prime} \text { ber., } \quad n=12 \\
& m: r=(110):(101) \quad 5558 \quad 5550 \frac{1}{6} \quad 3
\end{aligned}
$$

Keine Spaltbarkeit konnte wahrgenommen werden; die Corrosionen sind mit dem monosymmetrischen Systeme im Einklange. Auf den Prismenflächen findet die Auslöschung etwa $30^{\circ}$ von der Kante $m: m$ nach aufwärts gegen die Flächen $q$ statt. Die Ebene der optischen Axen ist (010). Auf einer Platte, die ungefähr parallel der Ebene (100) geschliffen war, sieht man den Austritt der ersten Bisectrix a. Der scheinbare Axenwinkel $2 E$ beträgt etwa $72^{\circ}$ (im Konoskope bei Natriumlicht gemessen).

Spec. Gewicht (in Acetylentetrabromid) =2,128.

\section{Kalium- und Ammoniummolybdänhexarhodanacetat} $\mathrm{Mo}(\mathrm{CNS})_{6}\left(\mathrm{H}_{2} \mathrm{O}\right) \mathrm{K}_{3} . \mathrm{CH}_{3} \mathrm{CO}_{2} \mathrm{H}$. Dargestellt von Johanna Maas und J. Sand, Ber. d. d. chem. Ges. 1908, 41, 1509, 3372.

Rhombisch pyramidal. $a: b: c=0,7124: 1: 0,6318$.

1) Zeitschr. f. anorg. Chemie $1906,50,28$. 
Rotbraune, wenig durchsichtige Krystalle der Combination $m\{110\}$, $a\{100\}, q\{011\}, *\{01 \bar{T}\}, r\{101\}$. Die vorherrschend ausgebildeten Prismenflächen $m$ sind meist im Gleichgewicht; $a$ tritt teils als schmale Facette, teils aber auch als recht breite Fläche auf. Von den Endflächen ist meist nur $q$ vorhanden; seltener auch noch die kleinen Flächen von $r$, die aber immer nur an einem Pole auftreten (Fig, 15). Ein einziger Krystall zeigte zwischen $m$ und $q$ schmale undeutliche Kantenabstumpfungen. Die nur angenähert möglichen Messungen deuten auf die Indices $\{121\}$ resp. $\{231\}$. Häufig sind Zwillinge nach $(01 \bar{T})$. Auf Krystallen mit $a$-Flächen ist infolge der Streifung $\|$ [001] die Zwillingsbildung deutlich zu erkennen (Fig. 16).

Fig. 15.

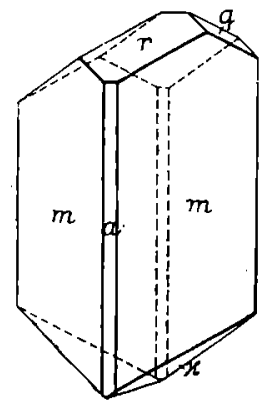

Fig. 16 .

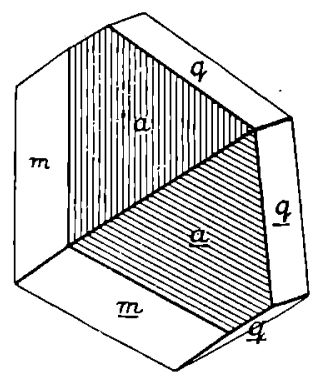

Fig. 17.

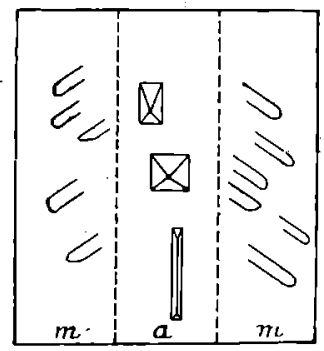

Die Messung wurde durch auf fast allen Flächen vorhandene Vicinalflächen sehr erschwert.

$$
\begin{aligned}
& \text { Berechnet: Beobachtet: }
\end{aligned}
$$

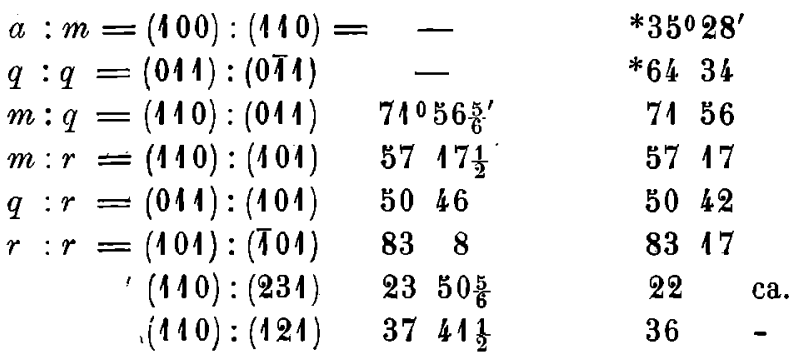

Auf $a$ entstehen mit Wasser leicht Ätzfiguren, die nach $\{010\}$, nicht aber nach $\{001\}$ symmetrisch sind; auf den Flächen von $m$ liegen die Ätzfiguren symmetrisch zu $\{100\}$ bezw. $\{010\}$ (Fig. 17).

Die Ebene der optischen Axen ist $\{010\}$.

Pleochrö̈smus: || der Verticalaxe bräunlichrot | beobachtet auf den $\perp$ zur Verticalaxe bräunlichgelb $m$-Flächen.

Spaltbarkeit deutlich nach $\{110\}$.

Spec. Gewicht (in Acetylentetrabromid) 1,893. 


$$
\mathrm{Mo}(\mathrm{CNS})_{6} \mathrm{H}_{2} \mathrm{O}\left(\mathrm{NH}_{4}\right)_{3} . \mathrm{CH}_{3} \mathrm{CO}_{2} \mathrm{H} \text {. }
$$

Dargestellt von J. Maas und J. Sand, Ber. d. d. chem. Ges. 1908,

$$
\text { 40, 4504;41, } 1500 .
$$

Rhombisch pyramidal. $a: b: c=0,7255: 1: 0,6338$. Die mit dem Kaliumsalze isomorphen Krystalle zeigen die Combination $m\{110\}, q\{011\}$, $x\{01 T\}, r\{101\}, \varrho\{10 \bar{T}\}$. Der Krystallhabitus ist dicktafelig nach einem Paar Flächen von $m$ und zugleich gestreckt nach der Verticalaxe. Von den Endflächen ist an einem Pole $r$ groß und $q$ klein, am anderen $x$ groß und $\varrho$ klein ausgebildet (Fig. 18).

Fig. 18.

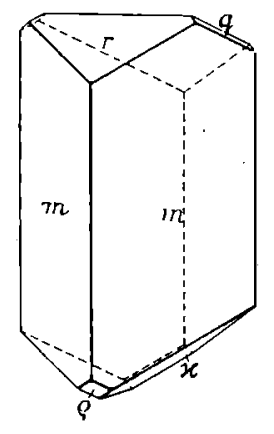

$$
\begin{aligned}
& r: r=(101):(\text { T01) } \\
& q: q=(011):(0 \overline{1} 1) \\
& m: m=(110):(1 \overline{1} 0) \\
& m: q=(110):(011) \\
& m: r=(110):(101) \\
& r: q=(101):(011)
\end{aligned}
$$

Fig. 19.

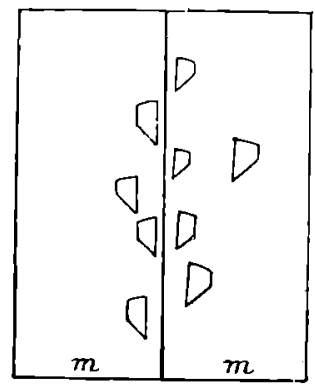

Berechnet:

\section{Beobachtet:}

*82017'

*64 44

7150

$71 \quad 40$

$57 \quad 49$

$50 \quad 28$

Mit Wasser erhält man auf den $m$-Flächen leicht Ätzfiguren, welche unsymmetrisch nach $\{001\}$, symmetrisch gegen die Kante $m: m$ sind (Fig. 19).

Die Ebene der optischen Axen ist $\{010\}$.

Pleochroïsmus: || der Verticalaxe bräunlichgelb,

$$
1 \text { - - grünlichgelb. }
$$

Spaltbarkeit unvollkommen nach $\{110\}$.

Spec. Gewicht (in Acetylentetrabromid) 1,654. 